\title{
Dietary Intake of Vitamin D Is Related to Blood Levels of Advanced Glycation End Products during a Weight Loss Program in Obese Women
}

\author{
Shin Sukino ${ }^{1}$, Kazuhiko Kotani ${ }^{1,2,3}$, Shinsuke Nirengi ${ }^{1}$, Alejandro Gugliucci ${ }^{3}$, Russell Caccavello ${ }^{3}$, Kokoro \\ Tsuzaki ${ }^{1}$, Yaeko Kawaguchi ${ }^{1}$, Kaoru Takahashi ${ }^{4}$, Kahori Egawa ${ }^{5}$, Hiroshi Shibata ${ }^{5}$, Makiko Yoshimura6, \\ Yoshinori Kitagawa7, Naoki Sakane ${ }^{1 凶}$ \\ 1. Division of Preventive Medicine, National Hospital Organization Kyoto Medical Center, Kyoto, Japan; \\ 2. Division of Community and Family Medicine, Jichi Medical University, Shimotsuke-City, Tochigi, Japan; \\ 3. Glycation, Oxidation and Disease Laboratory, Touro University-California, CA, USA; \\ 4. Hyogo Health Service Association; \\ 5. Institute for Health Care Science, Health Care Science Center, Suntory Wellness Limited, Kyoto, Japan; \\ 6. HE Center, R\&D Support Division, Suntory Business Expert Limited, Kyoto, Japan; \\ 7. Safety Science Institute, Quality Assurance Division, Suntory Business Expert Limited, Kyoto, Japan.
}

$\triangle$ Corresponding author: Naoki Sakane, MD, PhD. Division of Preventive Medicine, Clinical Research Institute, National Hospital Organization Kyoto Medical Center, 1-1 Mukaihata-cho, Fukakusa, Fushimi-ku, Kyoto 612-8555, Japan. Tel: +81-75-641-9161 Fax: +81-75-645-2781 E-mail: nsakane@kyotolan.hosp.go.jp.

(c) Ivyspring International Publisher. This is an open access article distributed under the terms of the Creative Commons Attribution (CC BY-NC) license (https://creativecommons.org/licenses/by-nc/4.0/). See http://ivyspring.com/terms for full terms and conditions.

Received: 2016.06.16; Accepted: 2016.07.06; Published: 2016.07.15

\begin{abstract}
BACKGROUND/OBJECTIVES: Advanced glycation end products (AGEs) contribute to the pathophysiology of lifestyle-related diseases. To identify nutrients associated with AGEs, this study explored the factors by investigating the relationship between nutrients changes and changes of blood AGEs during a weight loss program in obese women.

SUBJECTS/METHODS: Twenty-five obese women (age: $50 \pm 8$ years, body mass index: $28.7 \pm$ $3.4 \mathrm{~kg} / \mathrm{m}^{2}$ ) underwent a weight loss program with energy-restricted meal replacement for 2 months. Three-day weighted dietary records and blood tests including blood AGEs were performed at the baseline and after the 2-month intervention. Their correlation was examined during the intervention period.
\end{abstract}

RESULTS: The changes in AGEs were significantly and negatively correlated with those of intake levels of vitamin $D(r=-0.54 ; P<0.05)$.

CONCLUSION: Vitamin $D$ might be a useful nutrient to reduce AGEs in obese women.

Key words: AGEs, weight loss, vitamin D.

\section{Introduction}

Advanced glycation end products (AGEs), formed by the Maillard reaction, contribute to the development of lifestyle-related diseases $[1,2,3]$. Therefore, the regulation of AGEs is crucial, and various environmental factors, such as dietary factors, can act to regulate them $[4,5,6,7]$. However, the dietary factors affecting AGEs during a weight loss program in subjects without diabetes are unknown. Additionally, focusing only on calorie intake during weight loss is known to lead to unsatisfactory nutrient status. Thus, it is important to examine the nutrients affecting AGEs during weight loss. 
Previously, we reported a significant reduction in blood AGEs throughout a weight loss program using energy-restricted meal replacement [8]; however, it did not include an analysis that explored nutrients related to blood AGEs. Therefore, the purpose of the current study was to explore nutrients in relation to changes of blood AGEs among obese women as an additional analysis of that study on weight loss [8].

\section{Subjects and Methods}

\section{Study design}

We conducted exploratory research to identify possible nutrients related to blood AGEs. The subjects improved their dietary habits by consuming an energy-restricted meal replacement instead of their regular dinner every day for 2 months, as previously described [8]. The study conformed to the ethical guidelines of the 2013 Declaration of Helsinki and was approved by the Ethics Committee of Kyoto Medical Center (Approval No. 06-28).

\section{Subjects}

The current study involved subjects in whom the investigation of nutrients was completely unrestricted. Because nutrient intake might have different influences on clinical outcomes based on the body mass index (BMI), the study involved subjects with certain homogeneous characteristics (female and obese [BMI of $\geq 25 \mathrm{~kg} / \mathrm{m}^{2}$ ]) [9]. Thus, 25 obese women (mean age: $50.8 \pm 8.3$ [standard deviation] years, mean BMI: $28.7 \pm 3.4 \mathrm{~kg} / \mathrm{m}^{2}$ ) were analyzed in the current study.

\section{Diets}

The women consumed an energy-restricted meal replacement that contained 5,023 kJ/day, which reduced their caloric intake by approximately $20 \%$ per day, $40 \%$ carbohydrate, $7 \%$ fat, and $47 \%$ protein (Diet's TM, Suntory CO., Ltd., Osaka, Japan) [8], every day for 2 months for dinner. A registered dietitian instructed each subject on planning a nutritionally balanced and constant diet for their other meals, and compliance was confirmed through daily dietary records [8].

\section{Measurements}

We measured the following parameters at the baseline and after 2 months of intervention. Height, body weight, and BMI were measured. Blood pressure was measured using a mercury sphygmomanometer. Plasma glucose, total cholesterol, triglyceride, and high- and low-density lipoprotein cholesterol (LDL-C) levels were determined using enzymatic methods. Blood AGEs were measured based on the fluorescence intensity recorded at $440 \mathrm{~nm}$, upon excitation at $350 \mathrm{~nm}$, using a Spectramax Gemini XPS spectrofluorometer with Softmax Pro software (Molecular Devices, Sunnyvale, California, USA). Average daily energy and nutrient intake was estimated by a registered dietitian from 3-day weighted-food records using Healthy Maker Pro501 (Mushroomsoft Co., Ltd., Okayama, Japan). Detailed instructions on the use of scales to accurately weigh food and drink and record consumption, including leftovers, were provided to the subjects.

\section{Statistical analyses}

Data are expressed as means \pm standard deviation. The differences between the baseline values and those after intervention were analyzed using the paired t-test. For a simple correlation analysis, the Pearson correlation coefficient was used to determine correlations between changes in nutrients and those of blood AGEs. A multiple regression analysis model for changes in vitamin D intake (dependent variable) examined the independence of changes in AGEs, with adjustments made for age and changes in body weight (basic confounders) or the previous two plus changes in dietary fiber intake (a significant factor correlated with changes in AGEs in the simple correlation analysis). All statistical analyses were performed using SPSS program (IBM SPSS 23.0, Tokyo, Japan). Values were considered significant at $P$ $<0.05$.

\section{Results}

\section{Subject characteristics}

The data at baseline and after intervention are presented in Table 1. After intervention, significant reductions in weight, blood pressure, plasma glucose, LDL-C, and AGEs were observed (these results are consistent with those of our previous report [8]).

\section{Nutrients at baseline and after intervention}

The data on nutrients at baseline and after intervention are presented in Table 2. After intervention, significant reductions in energy, protein, total fat, carbohydrate, vitamin A, thiamin, and salt intake were observed $(P<0.05)$.

\section{Relationships between changes in dietary factors and changes in AGEs}

Changes in blood AGEs were significantly and inversely correlated with changes in vitamin $\mathrm{D}$ and dietary fiber $(P<0.05)$. A model adjusted for age and changes in body weight depicted that this correlation was independent of these confounders $(\beta=-0.49, P<$ 
0.05). An additional model adjusted for the previous variables as well as changes in dietary fiber intake also depicted this independence $(\beta=-0.47, P<0.05)$.

\section{Discussion}

The current study (as an additional analysis of our previous study [8]) revealed that changes in vitamin $\mathrm{D}$ were negatively correlated with those of blood AGEs, which was independent of those of weight loss among obese women, during the weight loss period. Recently, vitamin D has been indicated to exhibit anti-glycation $[10,11,12]$ and anti-oxidation (oxidative stress is the final step of the Maillard reaction) effects $[13,14]$. In humans, there have been only two AGE studies using high-dosage vitamin D $[10,12]$. Vitamin D supplementation $(1,250 \mu \mathrm{g}$ once weekly) reportedly increased serum soluble receptors for AGEs (sRAGE) [12], which act as decoys by binding to blood AGEs. A vitamin D-fortified (25 $\mu \mathrm{g} / 500 \mathrm{~mL}$ ) yogurt drink decreased blood AGEs after 3 months [10]. Therefore, the results of this study confirmed the previous findings under free feeding during weight loss.

Table 1. Baseline characteristics, those after intervention.

\begin{tabular}{llll}
\hline Variables & Baseline & After intervention & Change \\
\cline { 2 - 4 } & mean (SD) & $-4.6(1.9)^{*}$ \\
\hline Weight (kg) & $69.7(8.1)$ & $65.1(7.9)$ & $-5.4(10.4)^{*}$ \\
SBP (mmHg) & $129.8(15.9)$ & $124.4(15.0)$ & $-5.6(7.2)^{*}$ \\
DBP (mmHg) & $81.1(11.5)$ & $75.5(10.5)$ & $-0.26(0.36)^{*}$ \\
Plasma glucose (mmol/L) & $5.06(0.43)$ & $4.80(0.39)$ & $-0.14(0.38)$ \\
Triglycerides (mmol/L) & $1.02(0.47)$ & $0.88(0.40)$ & $-0.07(0.18)$ \\
HDL-cholesterol (mmol/L) & $1.68(0.37)$ & $1.61(0.31)$ & $-0.31(0.30)^{*}$ \\
LDL-cholesterol (mmol/L) & $3.35(0.69)$ & $3.04(0.71)$ & $-4.7(5.6)^{*}$ \\
AGEs (AU) & $61.0(9.0)$ & $56.2(8.8)$ & \\
\hline
\end{tabular}

Data are mean (standard deviation)

SBP: systolic blood pressure, DBP: diastolic blood pressure, HDL: high-density lipoprotein, LDL: low-density lipoprotein, AGEs: advanced glycation end products.

The differences between the baseline and after intervention were analyzed using the t-test. ${ }^{*} P<0.05$.

Table 2. Baseline nutrients, those after intervention, changes, and correlation between changes in nutrients and changes in AGEs.

\begin{tabular}{|c|c|c|c|c|}
\hline \multirow[t]{2}{*}{ Nutrients } & Baseline & After intervention & Change & \multirow{2}{*}{$\begin{array}{l}\text { Correlation coefficient } \\
\mathrm{r}\end{array}$} \\
\hline & mean $(\mathrm{SD})$ & & & \\
\hline Energy (kcal) & $2,098.9(454.6)$ & $1,683.1(461.1)$ & $-415.8(382.5)^{*}$ & -0.16 \\
\hline Protein (g) & 79.7 (17.5) & $71.1(17.5)$ & $-8.6(16.5)^{*}$ & -0.17 \\
\hline Total fat (g) & $71.0(25.7)$ & $51.6(1.2)$ & $-19.4(21.6) *$ & -0.11 \\
\hline Carbohydrate (g) & $276.5(56.9)$ & $231.4(70.1)$ & $-45.1(56.1)^{*}$ & -0.16 \\
\hline Dietary fiber (g) & $15.8(4.2)$ & $17.0(7.5)$ & $1.2(8.8)$ & $-0.40 *$ \\
\hline Vitamin A $(\mu \mathrm{g})$ & $643.2(459.6)$ & $564.0(249.5)$ & $-79.2(494.3)$ * & -0.10 \\
\hline Vitamin D $(\mu \mathrm{g})$ & $8.5(6.6)$ & $7.8(5.1)$ & $-0.7(7.7)$ & -0.54 * \\
\hline Vitamin E (mg) & $7.8(1.8)$ & $7.6(2.6)$ & $-0.1(3.2)$ & -0.07 \\
\hline Vitamin K $(\mu \mathrm{g})$ & $272.8(138.6)$ & $248.6(144.0)$ & $-24.2(182.4)$ & -0.38 \\
\hline Thiamin (mg) & $1.1(0.4)$ & $1.0(0.3)$ & $-0.2(0.4)$ * & -0.05 \\
\hline Riboflavin (mg) & $1.3(0.3)$ & $1.2(0.4)$ & $-0.1(0.4)$ & -0.23 \\
\hline Vitamin B6 (mg) & $1.4(0.3)$ & $1.3(0.6)$ & $0.0(0.6)$ & -0.39 \\
\hline Vitamin B12 $(\mu \mathrm{g})$ & $7.6(5.0)$ & $8.1(5.5)$ & $0.5(6.7)$ & -0.02 \\
\hline Vitamin C (mg) & $115.9(47.8)$ & $122.0(51.9)$ & $6.1(57.6)$ & -0.33 \\
\hline Salt (g) & $10.6(2.1)$ & $9.7(2.3)$ & $-0.9(2.2) *$ & 0.13 \\
\hline Calcium (mg) & $576.2(189.1)$ & $683.2(529.9)$ & $107.0(519.0)$ & -0.04 \\
\hline Iron (mg) & $8.5(2.0)$ & $8.5(3.2)$ & $0.0(3.1)$ & -0.32 \\
\hline
\end{tabular}

Data are mean (standard deviation) or correlation coefficient.

The differences between the baseline and after intervention were analyzed using the t-test. As a simple correlation analysis, the Pearson product-moment correlation coefficient was used for the relationship between the changes in nutrients and those of blood AGEs. ${ }^{*} P<0.05$. 
In this study, we could not identify food variables that affect AGEs. Vitamin D is mainly included in mushrooms [15] and shellfish [16] in food variables. In a partial correlation analysis, we found a negative correlation between mushroom ingestion and changes in AGEs after adjustment for weight loss $(P=0.06)$ (data not shown). Further studies are needed to investigate whether mushrooms can be a potential source of vitamin $\mathrm{D}$ to reduce AGEs. There were certain limitations to the current study, namely a relatively small sample size and the blood vitamin D concentration was not evaluated.

In conclusion, the changes in vitamin D were negatively correlated with those of blood AGEs, independent of the changes of weight loss, during a weight loss period among obese women. Further studies are warranted to support our results.

\section{Acknowledgments}

This work was in part supported by Suntory, JSPS KAKENHI Grant Number 26350178, Hokuto Foundation for Bioscience, and the Japanese Council for Science, Technology and Innovation (CSTI), SIP (Project ID 14533567), "Technologies for creating next-generation agriculture, forestry and fisheries" (funding agency: Bio-oriented Technology Research Advancement Institution, NARO).

\section{Competing Interests}

The authors have declared that no competing interest exists.

\section{References}

1. Müller MJ, Lagerpusch M, Enderle J, et al. Beyond the body mass index: tracking body composition in the pathogenesis of obesity and the metabolic syndrome. Obes Rev. 2012; 2: 6-13.

2. Kilhovd BK, Juutilainen A, Lehto $S$, et al. Increased serum levels of advanced glycation endproducts predict total, cardiovascular and coronary mortality in women with type 2 diabetes: a population-based 18year follow-up study. Diabetologia. 2007; 50: 1409-17.

3. Uribarri J, del Castillo MD, de la Maza MP, et al. Dietary advanced glycation end products and their role in health and disease. Adv Nutr. 2015; 6: 461-73.

4. Uribarri J, Woodruff S, Goodman S, et al. Advanced glycation end products in foods and a practical guide to their reduction in the diet. J Am Diet Assoc. 2010; 110: 911-916.

5. Uribarri J, Cai W, Sandu O, et al. Diet-derived advanced glycation end products are major contributors to the body's AGE pool and induce inflammation in healthy subjects. Ann NY Acad Sci. 2005; 1043: 461-6.

6. Uribarri J, Cai W, Peppa M, et al. Circulating glycotoxins and dietary advanced glycation endproducts: two links to inflammatory response, oxidative stress, and aging. J Gerontol A Biol Sci Med Sci. 2007; 62: 427-33.

7. Wellner A, Huettl C, Henle T. Formation of Maillard reaction products during heat treatment of carrots. J Agric Food Chem. 2011; 59: 7992-8.

8. Gugliucci A, Kotani K, Taing J, et al. Short-term low calorie diet intervention reduces serum advanced glycation end products in healthy overweight or obese adults. Ann Nutr Metab. 2009; 54: 197-201.

9. Sasazuki S, Inoue M, Tsuji I, et al. Body mass index and mortality from all causes and major causes in Japanese: results of a pooled analysis of 7 large-scale cohort studies. J Epidemiol. 2011; 21: 417-30.
10. Nikooyeh B, Neyestani TR, Tayebinejad N, et al. Daily intake of vitamin D- or calcium-vitamin D-fortified Persian yogurt drink (doogh) attenuates diabetes-induced oxidative stress: evidence for antioxidative properties of vitamin D. J Hum Nutr Diet. 2014; Suppl 2: 276-83.

11. Salum E, Kals J, Kampus P, et al. Vitamin D reduces deposition of advanced glycation end-products in the aortic wall and systemic oxidative stress in diabetic rats. Diabetes Res Clin Pract. 2013; 100: 243-9.

12. Irani M, Minkoff $H$, Seifer DB, et al. Vitamin D increases serum levels of the soluble receptor for advanced glycation end products in women with PCOS. J Clin Endocrinol Metab. 2014; 99: E886-90.

13. Polidoro L, Properzi G, Marampon F, et al. Vitamin D protects human endothelial cells from $\mathrm{H}_{2} \mathrm{O}_{2}$ oxidant injury through the Mek/Erk-Sirt1 axis activation. J Cardiovasc Transl Res. 2013; 6: 221-31.

14. Bodiga VL, Eda SR, Bodiga S. Advanced glycation end products: role in pathology of diabetic cardiomyopathy. Heart Fail Rev. 2014; 19: 49-63.

15. Chen SY, Yu HT, Kao JP, et al. Consumption of vitamin D2 enhanced mushrooms is associated with improved bone health. J Nutr Biochem. 2015; 26: 696-703.

16. Liu J, Arcot J, Cunningham J, et al. New data for vitamin D in Australian foods of animal origin: impact on estimates of national adult vitamin D intakes in 1995 and 2011-13. Asia Pac J Clin Nutr. 2015; 24: 464-71. 